\title{
Selective ECR heating of trapped/passing electrons in the W7-X stellarator
}

\author{
N. B. Marushchenko, V. Erckmann, C. D. Beidler, J. Geiger, H. P. Laqua, \\ P. Helander, H. Maassberg, Y. Turkin \\ Max Planck Institute for Plasma Physics, EURATOM Association, \\ Wendelsteinstr. 1, D-17491 Greifswald, Germany
}

\begin{abstract}
Using specific features of the magnetic equilibrium in the W7-X stellarator, ECRH scenarios with X2 and X3 modes are discussed. The aim is to explore the possibility of selective heating of the different classes of electrons, passing and trapped, by different RF beams with different frequencies, which can be launched from ports located in different crosssections of the device. Perspectives for this kind of experiments in W7-X are estimated numerically by coupling transport and ray tracing codes.
\end{abstract}

Keywords: plasma, stellarators, electron cyclotron resonance heating, trapped particles PACS: $52.25 . \mathrm{Os}, 52.50 .-\mathrm{b}, 52.50 . \mathrm{Sw}, 52.55 . \mathrm{Hc}$

\section{INTRODUCTION}

The main scientific goal of the W7-X stellarator [1] is to demonstrate good plasma confinement with steady-state operation with reactor-relevant parameters. The magnetic equilibrium (a low-shear configuration of the Helias type with five field periods) is quite flexible and well optimized with respect to low neoclassical transport (the "standard" configuration, with the variation of magnetic field along the axis $B_{\max } / B_{\min }=1.09$ ) and small bootstrap current (the "high-mirror" configuration, with $B_{\max } / B_{\min }=1.22$ ) [2].

The system for electron cyclotron resonance heating (ECRH) in W7-X has been designed for continuous operation with total injected power up to $10 \mathrm{MW}$. The ECRH system contains ten gyrotrons, which generate waves at $140 \mathrm{GHz}$ or (optionally) $105 \mathrm{GHz}$ with a power up to $1 \mathrm{MW}$ and $0.5 \mathrm{MW}$ each, respectively [3, 4]. The main ECRH scenarios are designed for operation at $B_{0} \simeq 2.5 \mathrm{~T}$ and $140 \mathrm{GHz}$ with the X2-mode $\left(n_{e}<1.2 \times 10^{20} \mathrm{~m}^{-3}\right)$ or O2-mode $\left(n_{e}<2.4 \times 10^{20} \mathrm{~m}^{-3}\right)$. The launch-ports are situated near the "bean-shaped" poloidal plane, where the the maximum of $B$ is located, which minimises the direct heating oftrapped electrons. Due to the rapid redistribution over the fluxsurface, the power density absorbed by passing electrons is sufficiently low that a linear treatment is expected to be appropriate, and, furthermore, conventional neoclassical transport theory should be valid.

However, there is a number of experiments (see for example [5-8]) as well as theoretical investigations [6, 7, 9, 10] which indicate significant kinetic effects for the transport in the case when a substantial fraction of the RF power is absorbed by trapped electrons. This can be especially important for stellarators, where the trapped electrons, being locked within the toroidal ripples, do not spread the absorbed power toroidally over the magnetic surface, and in the poloidal direction only do so by their precessional motion. Then, the local absorbed power density is significantly increased, and quasilinear (or even non-linear [11]) effects may be important for the absorption modelling. As a consequence, the generation of additional convective losses [9] can be observed. These features are still inadequately explored, and both experimental and theoretical research in this direction is important.

In order to study the kinetic effects induced by the direct heating of trapped electrons in W7-X, two additional ports near the "triangular" poloidal plane have been reserved. Here, the minimum of $B$ is located and, in contrast to the "bean-shaped" plane, the radial gradient of $B$ is small and can even change sign along the sight-line. For the RF beam launched here, trapped electrons can play a significant role in the cyclotron absorption. The RF power is injected using the "remote steering launcher" which allows for simultaneous switching between the directions and does not contain any moving parts [12].

The present work is devoted to study of the ECRH scenarios in W7-X at reduced magnetic field, $B_{0} \simeq 1.85 \mathrm{~T}$, with both $105 \mathrm{GHz}$ (X2-mode) and $140 \mathrm{GHz}$ (X3-mode) injected into the plasma from the different ports. While the $\mathrm{X} 2$-mode launched through the max of $B$ is absorbed only by passing electrons, the power fraction from the X3-mode 

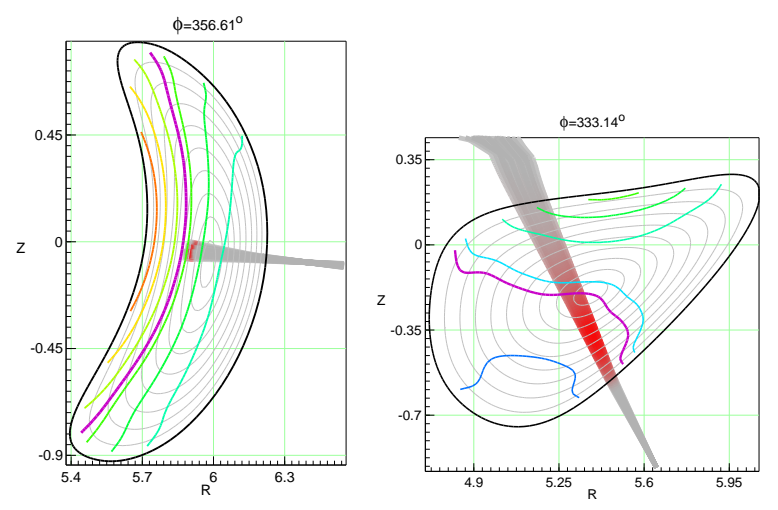

FIGURE 1. Projections of the RF beams onto the RZ cross-sections are shown. The toroidal angle of the cross-section corresponds to where the deposition is maximal. $B$-isolines together with the resonance value (magenta) are shown. The absorption is shown in red. Left: the beam at $105 \mathrm{GHz}$ (X2-mode) launched close to the "bean-shaped" plane. In the absorbtion zone $N_{\|} \simeq-0.25$. Right: the beam at $140 \mathrm{GHz}$ (X3-mode) launched near the "triangular" plane. In absorbtion zone $N_{\|} \simeq 0.6$.

absorbed by trapped electrons can be varied by the launch angle practically from zero to almost complete absorption. Simulations have been performed by the transport code [13] coupled with the ray tracing code TRAVIS [14]. Only the "standard" magnetic configuration has been applied, while the "high-mirror" configuration is found to be not well suitable for this topic. The present understanding and all theoretical assumptions adopted for modeling are based on the substantial experience from W7-AS and other experiments $[6-8,15]$.

\section{CONTROL OF POWER FRACTION ABSORBED BY TRAPPED ELECTRONS}

In the simulations, neoclassical core confinement with empirical anomalous transport at the plasma edge is assumed. The anomalous diffusivity is taken to scale inversely with the plasma density in the region of high density gradient and to decay exponentially towards the plasma axis. The radial electric field, electron and ion temperatures are advanced self-consistently with the calculation of the power deposition profiles by ray-tracing. The shape of the density profile with a gradient region of about $10 \mathrm{~cm}$ is fixed. The calculated electron temperature profile (not shown here) is flat within the normalised radius $\rho<0.28$, with $T_{e}(0) \simeq 5.1 \mathrm{keV}\left(T_{i}(0) \simeq 2.4 \mathrm{keV}\right)$.

Since the fraction of power absorbed by trapped electrons from the X3 mode injected near the "triangular" plane is sensitive to the location of the resonance, it is also sensitive to the plasma $\beta$-value. The modelling is performed for the "standard" magnetic configuration with $\langle\beta\rangle=2 \%$ (this value is consistent with the transport calculations which give $\langle\beta\rangle \simeq 2.5 \%)$. The magnetic field with $B_{\max }(0)=1.768 \mathrm{~T}$ and $B_{\min }(0)=1.663 \mathrm{~T}$ guarantees appropriate operation for both $\mathrm{X} 2$ at $105 \mathrm{GHz}$ and $\mathrm{X} 3$ at $140 \mathrm{GHz}$.

In the simulations, eight $105 \mathrm{GHz}$ beams of X2-mode with $0.5 \mathrm{MW}$ each are launched close to the "bean-shaped" plane. The plasma density is assumed to be constant, $n_{e}(0)=0.5 \times 10^{20} \mathrm{~m}^{-3}$ (the cut-off for X2-mode at this frequency is $0.68 \times 10^{20} \mathrm{~m}^{-3}$ ), making plasmas with $T_{e}>1 \mathrm{keV}$ optically thick with highly localized deposition profiles. For illustration, one X2-mode beam is show in Fig. 1 (left). The RF power is deposited in the central region but off-axis, since the resonance magnetic field is shifted to the high-field side. The obliqueness is not very large (in the absorption zone $N_{\|} \simeq-0.25$ ) and the Doppler shift is not significant. All the X2-beams have practically the same deposition profiles with complete single-pass absorption (total power $4 \mathrm{MW}$ ).

Two RF beams in X3-mode (140 GHz) with $1 \mathrm{MW}$ each are launched near the "triangular plane" using the "remote steering launcher". At the moment, two launch angles are considered most suitable for this kind of experiment, the "central line" and "+15 degree" directions, labelled with respect to orientation of the launcher. Both these angles correspond to rather high obliqueness, with $N_{\|} \simeq 0.6$ and $N_{\|} \simeq 0.4$, respectively. One example is shown in Fig. 1 (right), where the toroidal cross-section corresponds to the maximum of the deposition profile. For the X3-mode, the plasma is optically gray and single-pass absorption is switched by angles from $85 \%$ to $99 \%$.

By switching the launch conditions for the X3-beam near the "triangular plane" (minimum of $B$ ), one can change the fractions of power absorbed by trapped and passing electrons. This kind of selective heating of trapped or passing electrons is illustrated by Fig. 2, where the rate of absorption, $d P_{\mathrm{abs}} / d l$, decomposed into contributions from passing 

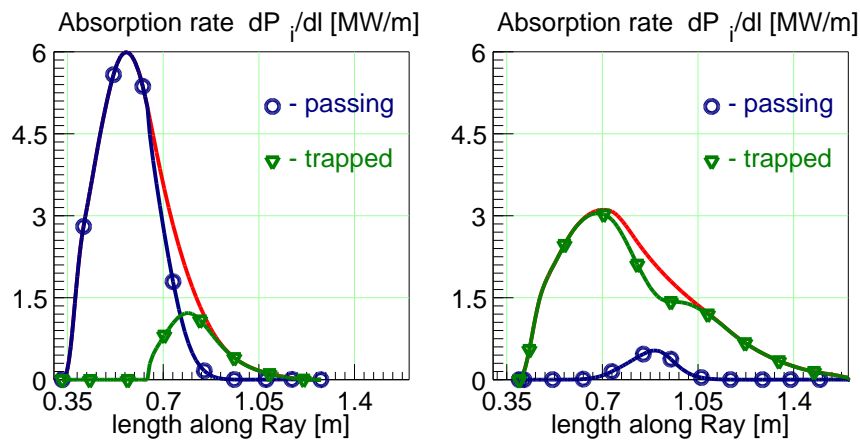

FIGURE 2. The rate of absorption, $d P_{\text {abs }} / d l$ along the central ray for the RF beam injected close to the "triangular" plane (140 GHz, X3-mode). Left: injection along "+15 degree" line (absorption by passing electrons dominates). Right: injection along the "central line" (absorption by trapped electrons dominates).

and trapped electrons are shown along the central ray for both directions, "+ 15 degree" and "central line". For both directions, which are very oblique with $N_{\|} \gtrsim 0.4$ and $N_{\|} \gtrsim 0.6$, respectively, the condition for cyclotron absorption, $n \omega_{c e} / \omega \geq\left(1-N_{\|}^{2}\right)^{1 / 2}$, is satisfied for $140 \mathrm{GHz}$ and $n=3$ practically for more than half the first ray path inside the plasma (see Fig. 1, right). Since the rate of absorption for X3 is roughly one order magnitude weaker than for X2mode, the width of the deposition profile is very broad (compare the length about $1 \mathrm{~m}$ with few $\mathrm{cm}$ for X2). Due to the weak $|\nabla B|$ along the ray, the normalized energy of resonant electrons responsible for the absorption varies only slightly along the ray trajectory, $E_{\text {res }} / T_{e} \sim 4$. The most important difference between the two directions is the location of the resonance line in momentum space. For the " +15 " direction, only the electrons with $u_{\|}<0$ are involved in the resonance, while for the "central line" the resonance line occupies both half-planes, $u_{\|} \leq 0$ and $u_{\|} \geq 0$.

In Fig. 3, the total deposition profile, $d P_{\text {abs }} / d V$, and its integral, $P_{\text {abs }}(\rho)=\int_{0}^{\rho} d P_{\text {abs }} / d V V^{\prime} d \rho$ are shown together with the contributions from passing and trapped electrons. This case corresponds to the launch of the RF-beam $(140 \mathrm{GHz}$, X3 mode) through the "remote steering launcher" along the "+15 degree" line. The deposition from the X2-mode is well localized in $0.17<\rho<0.4$ (only passing electrons contribute), while for the X3-mode the profile is very broad and non-negligible up to $\rho<0.8$. The contribution of the trapped electrons to the absorption is rather small: about $15 \%$ for the X3-mode and about 5\% from the total absorbed power (see Fig. 3, right).

For comparison, consider what happens when the launch direction of the X3-mode is changed to be along the "central line". Since the deposition profile of the $4 \mathrm{MW}, \mathrm{X} 2$-mode contribution hardly changes, the temperature profiles predicted by the transport code are practically the same. In Fig. 4, the total deposition and the integral power profiles are shown. One can see (left) that the shape and location of the deposition profile from X2-mode is the same as before, while the deposition from the X3-mode is shifted toward the axis. However, the trapped electrons absorb approximately 94\% from the X3-mode, that is about $28 \%$ of the total absorbed power (see Fig. 4, right).

The power densities shown in Figs. 3 and 4 are calculated under the assumption that the absorbed power is spread
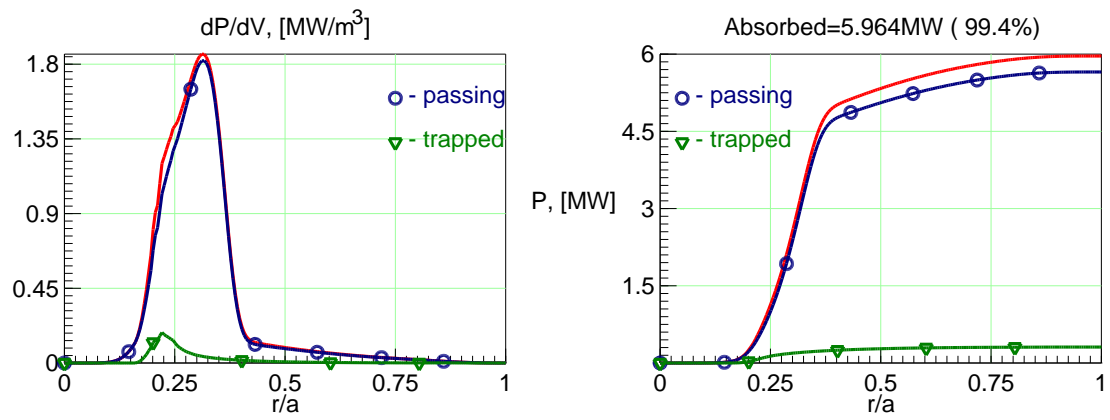

FIGURE 3. Total deposition profile and its integral for all RF beams with $P_{R F}^{\text {total }}=6 \mathrm{MW}$ are shown. Both the X2-mode (eight $0.5 \mathrm{MW}$ beams at $105 \mathrm{GHz}$ ) and the X3-mode (two $1 \mathrm{MW}$ beams at $140 \mathrm{GHz}$ ) have been completely absorbed. The fraction of power absorbed by trapped electrons from the X3-mode $P_{\mathrm{abs}}^{\text {trap }} / P_{\mathrm{abs}}^{\mathrm{total}} \simeq 5 \%$. 

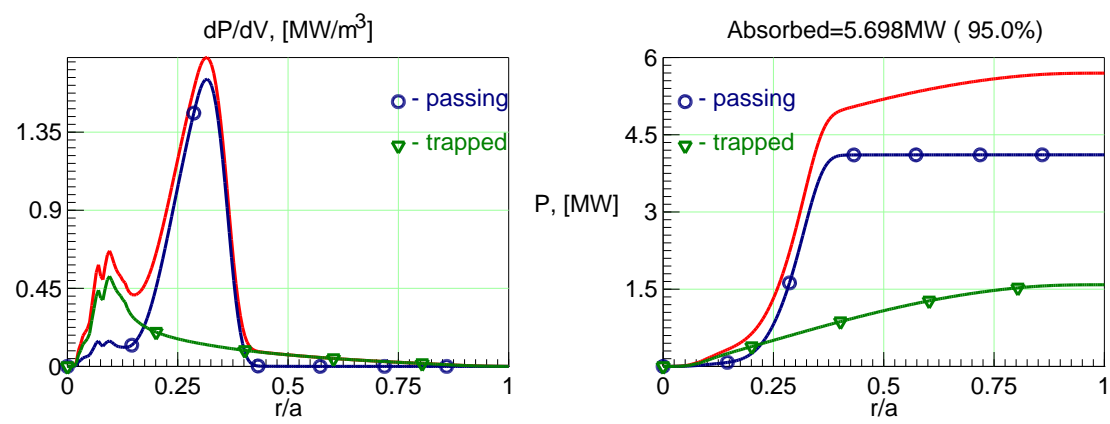

FIGURE 4. The same as in Fig. 2 with only the difference that two beams at $140 \mathrm{GHz}$ are injected along the "central line". The fraction of power absorbed by trapped electrons from X3-mode $P_{\mathrm{abs}}^{\text {trap }} / P_{\mathrm{abs}}^{\text {total }} \simeq 28 \%$.

over the entire flux surface. However, this is strictly true only for passing electrons. In contrast, the power density calculated by the code for the trapped electrons (Fig. 4, left) does not take into account the geometrical factor related to the reduced volume occupied by trapped electrons. In fact, the time required for passing electrons to return into the RF beam is much longer than the period for re-entrance for the trapped electrons, in particular those that are locked within toroidal ripples. As a consequence, the local power density in the cyclotron interaction can be icreased significantly, and quasilinear effects may play a non-negligible role. Such effects need to be studied with help of more sophisticated kinetic modeling.

\section{SUMMARY}

In this paper, preliminary results of numerical simulations of ECRH scenarios in the W7-X stellarator with selective heating of trapped/passing electrons by the X3-mode $(140 \mathrm{GHz})$ launched near the "triangular" plane (minimum of $B$ ) are presented. The X3-heating is supposed to be supported by X2-heating at $105 \mathrm{GHz}$. Calculations are performed by coupled transport and ray-tracing codes.

It has been shown that the power fraction absorbed by trapped electrons is very sensitive to the launch conditions. For example, when changing of the launch angle for the beam used for X3-heating by $15^{\circ}$, the power fraction absorbed by the trapped electrons increases from $15 \%$ to $94 \%$. The power density absorbed by particles trapped in toroidal ripples is increased by a factor of about five due to geometrical effects, implying that quasilinear (or even non-linear) effects in cyclotron interaction may take place. These predictions could be explored in experiments with power absorption by ripple-trapped electrons, leading to an additional convective contribution to the radial transport. Note also that, since the current drive is roughly proportional to the power fraction deposited into the passing electrons, the change of the driven current can be used as a diagnostic tool.

\section{REFERENCES}

1. C. Beidler et al., Fusion Technol. 17, 148 (1990).

2. H. Maassberg, W. Lotz, and J. Nührenberg, Phys. Fluids B 5, 3728 (1993).

3. V. Erckmann et al., Fusion Sci. Technol. 52, 291 (2007).

4. G. Dammertz et al., IEEE Trans. Plasma Sci. 34, 173 (2006).

5. V. Erckmann, and U. Gasparino, Plasma Phys. Control. Fusion 36, 1869 (1994).

6. M. Romé et al., Plasma Phys. Control. Fusion 39, 117 (1997).

7. H. Maassberg et al., Phys. Plasmas 7, 295 (2000).

8. M. Hirsch et al., Plasma Physics and Controlled Fusion 50, 053001 (2008).

9. S. Murakami et al., J. Plasma Fusion Res. Ser. 1, 122 (1998).

10. J. Seol, C. C. Hegna, and J. D. Callen, Phys. Plasmas 14, 082505 (2007).

11. R. Kamendje, S. V. Kasilov, W. Kernbichler, and M. F. Heyn, Phys. Plasmas 10, 75-97 (2003).

12. V. Erckmann et al., this conference: P2.06 (2013).

13. Yu. Turkin et al., in Proc. 34th EPS Conference on Plasma Phys. Warsaw, 2 - 6 July 2007 ECA Vol.31F, P-1.148 (2007), 2007.

14. N. B. Marushchenko et al., J. Plasma Fusion Res. Ser. 2, S1000 (2007).

15. V. Erckmann et al., Fusion Eng. Design 53, 365 (2001). 